PDES, SUBMANIFOLDS AND

AFFINE DIFFERENTIAL GEOMETRY

BANACH CENTER PUBLICATIONS, VOLUME 69

INSTITUTE OF MATHEMATICS

POLISH ACADEMY OF SCIENCES

WARSZAWA 2005

\title{
A NAIVE-TOPOLOGICAL STUDY OF THE CONTIGUITY RELATIONS FOR HYPERGEOMETRIC FUNCTIONS
}

\author{
MASAAKI YOSHIDA \\ Department of Mathematics, Kyushu University \\ Fukuoka 810-8560, Japan \\ E-mail: myoshida@math.kyushu-u.ac.jp
}

\begin{abstract}
When the parameters are real, the hypergeometric equation defines a Schwarz triangle. We study a combinatorial-topological property of the Schwarz triangle when the three angles are not necessarily less than $\pi$.
\end{abstract}

1. Introduction. Let $E(a, b, c)$ be the hypergeometric differential equation

$$
x(1-x) u^{\prime \prime}+(c-(a+b+1) x) u^{\prime}-a b u=0,
$$

where $a, b$ and $c$ are real parameters. Its Schwarz map (cf. [Yo]) is defined by

$$
s: X=\mathbf{C}-\{0,1\} \ni x \mapsto z=u_{1}(x): u_{2}(x) \in Z \cong \mathbf{P}^{1}:=\mathbf{C} \cup\{\infty\},
$$

where $u_{1}$ and $u_{2}$ are two linearly independent solutions of $E(a, b, c)$. The image of the upper half plane $X^{+}=\{x \in \mathbf{C}-\{0,1\} \mid \Im(x) \geq 0\}$ is called the Schwarz triangle T. If

$$
|1-c|<1, \quad|c-a-b|<1, \quad|b-a|<1,
$$

then the Schwarz triangle is indeed a triangle bounded by three arcs with angles $\mid 1-$ $c|\pi| c-a-b \mid, \pi$ and $|b-a| \pi$, in this order. But otherwise, the Schwarz triangle $T$ may be fairly complicated and it would cover the sphere $Z$ many times. In this paper, we study the Schwarz triangle $T$ in a naive topological manner. It is a bit surprising that no one has ever studied this fundamental problem seriously. As the reader will see, the result is indeed not so simple. To formulate our problem, we need to fix some notation.

In any case, as far as the parameters are real, the images of the intervals $(-\infty, 0),(0,1)$ and $(1,+\infty)$ under the restriction $\left.s\right|_{X^{+}}$of $s$ are (parts of) circles; we call these circles $C_{(-\infty, 0)}, C_{(0,1)}$ and $C_{(1,+\infty)}$, respectively. If $x$ tends to 0 in $X^{+}$then $s(x)$ converges; we

2000 Mathematics Subject Classification: Primary 33C05.

Key words and phrases: hypergeometric function, Schwarz's s-function, contiguity relations. The paper is in final form and no version of it will be published elsewhere. 
denote the limit by $s(0)$, which is an intersection point of $C_{(-\infty, 0)}$ and $C_{(0,1)}$. In the same way we define $s(1)$ and $s(\infty)$. The triangle with vertices $\{s(0), s(1), s(\infty)\}$ is called $D$. Note that the three vertices $s(0), s(1)$ and $s(\infty)$ are not necessarily ordered positively around $D$. In Figures $1, \ldots, 4$, they are ordered positively; we omit the corresponding figures when they are ordered negatively.

We assume for a while that each angle, which is by definition a non-negative real number, of $D$ is non-zero, and that the sum of the three angles of $D$ is greater than $\pi$. Then the three circles divide the sphere $Z$ into eight triangles $D, A, B, C, \bar{A}, \bar{B}, \bar{C}, \bar{D}$. The three triangles $A, B$ and $C$ are adjacent to $D$, and each has two vertices in common with $D: A$ has vertices $\{s(1), s(\infty)\}, B$ has vertices $\{s(\infty), s(0)\}, C$ has vertices $\{s(0), s(1)\}$. Each of the three triangles $\bar{A}, \bar{B}$ and $\bar{C}$ has one vertex in common with $D: \bar{A}$ has vertex $s(0), \bar{B}$ has vertex $s(1), \bar{C}$ has vertex $s(\infty)$. The last one $\bar{D}$ is away from the three vertices. See Figure 1.
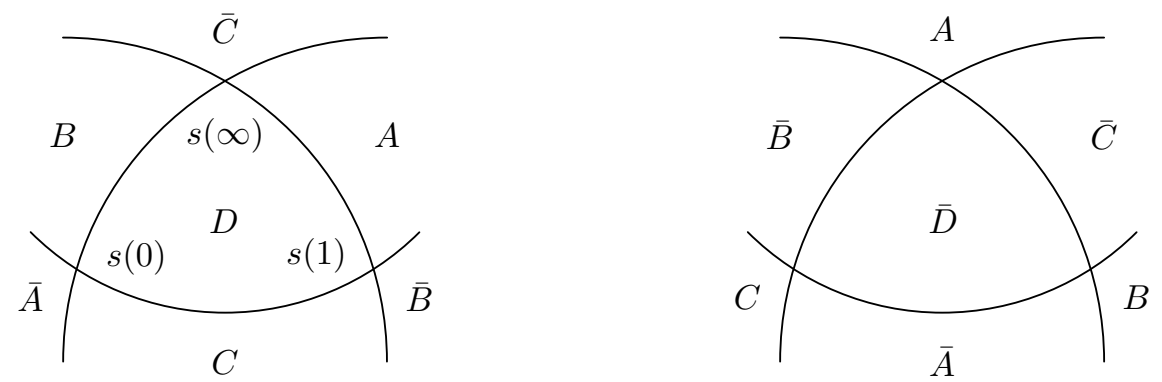

Fig. 1. Three circles bounding $D$, sum of the angles $>\pi$

As the sum of the three angles of $D$ tends to $\pi$, the triangle $\bar{D}$ degenerates to a point, which is the intersection of the three circles. See Figure 2.
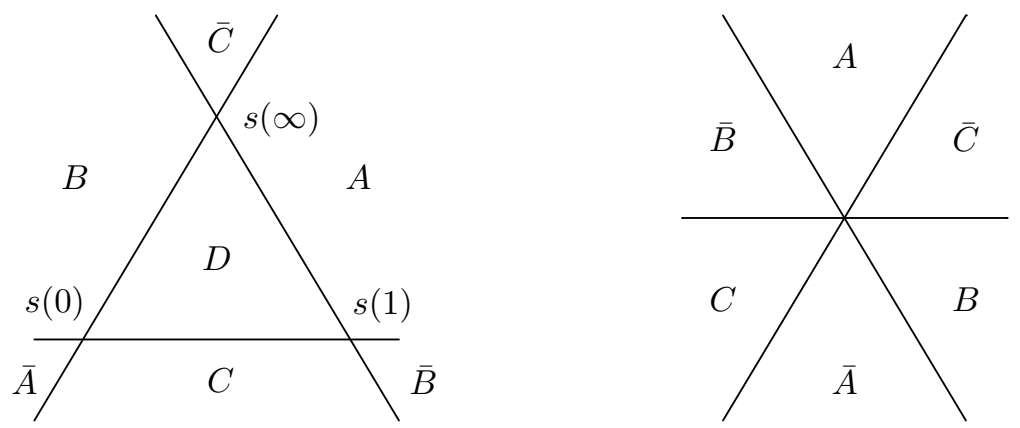

Fig. 2. Three circles bounding $D$, sum of the angles $=\pi$

As the sum of the three angles of $D$ becomes less than $\pi$, once degenerate intersection point recovers to be a triangle, which we call again $\bar{D} ; A, B$ and $C$ are now rectangles, and $\bar{A}, \bar{B}$ and $\bar{C}$ are biangles. See Figure 3 . 

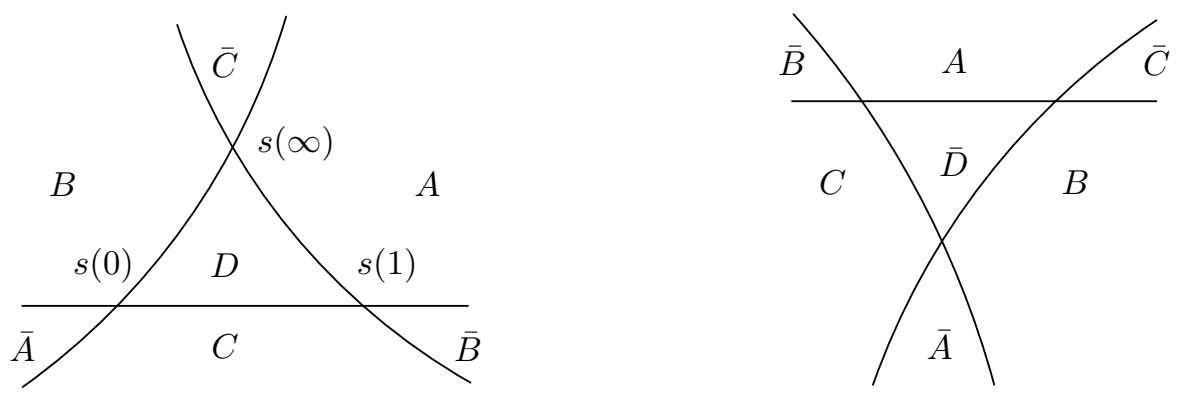

Fig. 3. Three circles bounding $D$, sum of the angles $<\pi$

When the angle of $D$ at, say, $s(0)$ is 0 , then the biangle $\bar{C}$ reduces to a point, and the rectangles $A$ and $B$ become triangles. In particular, when all the angles of $D$ are 0 , then the three biangles $\bar{A}, \bar{B}, \bar{C}$ disappear, and while $D$ and $\bar{D}$ remain to be triangles, $A, B$ and $C$ become biangles. See Figure 4 .

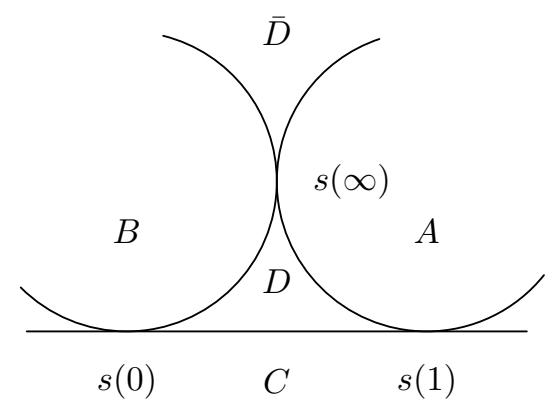

Fig. 4. Three circles bounding $D$ with zero angles

Now we are ready to formulate our problem. We wish to draw in a disk, identified with the upper half plane $X^{+}$, the inverse images under $\left.s\right|_{X^{+}}$of the domains $D, A, \ldots, \bar{C}, \bar{D}$ in $Z$. We call this drawing the chart $\mathbf{X}$. The chart tells us how the upper half plane $X^{+}$covers the target $Z$ under the Schwarz map $s$. We identify two charts if they are homeomorphic.

When we draw pictures, we assume that the sum of the angles of the triangle $D$ is greater than $\pi$. Other cases can be obtained from these by the deformation procedures described above.

Our strategy is as follows. We first find real numbers $\dot{a}, \dot{b}$ and $\dot{c}$ satisfying

$$
0 \leq 1-\dot{c}<1, \quad 0 \leq \dot{c}-\dot{a}-\dot{b}<1, \quad 0 \leq \dot{b}-\dot{a}<1,
$$

and

$$
\dot{a}-a, \dot{b}-b, \dot{c}-c \in \mathbf{Z} .
$$

If necessary, we exchange $a$ and $b$. Then such a triple $(\dot{a}, \dot{b}, \dot{c})$ exists uniquely. Let

$$
\dot{s}: X \rightarrow \dot{Z} \cong \mathbf{P}^{1}
$$

be the Schwarz map of the equation $E(\dot{a}, \dot{b}, \dot{c})$, and $\dot{T}$ its Schwarz triangle. Define circles 
$\dot{C}_{(-\infty, 0)}, \dot{C}_{(0,1)}, \dot{C}_{(0, \infty)}$ and triangles $\dot{D}, \dot{A}$, etc. as above. There is (Lemma in $\S 3$ ) a linear fractional transformation $\dot{Z} \rightarrow Z$ which takes the three circles $\dot{C}_{(-\infty, 0)}, \dot{C}_{(0,1)}, \dot{C}_{(0, \infty)}$ to the three circles $C_{(-\infty, 0)}, C_{(0,1)}, C_{(0, \infty)}$, respectively. The transformation does not necessarily send $\dot{D}$ to $D, \dot{A}$ to $A, \ldots$ Note that $\dot{D}=\dot{T}$ and that the vertices $\dot{s}(0), \dot{s}(1)$ and $\dot{s}(\infty)$ are ordered positively around $\dot{T}$. We study the Schwarz triangle $T$ by comparing with $\dot{T}$ through the map

$$
s \circ \dot{s}^{-1}: \dot{Z} \supset \dot{T} \rightarrow T \subset Z .
$$

(This map is studied also in [OY].) Note that $X^{+}$is homeomorphic to $\dot{T}$ under $\left.\dot{s}\right|_{X^{+}}$.

Let the parameters $(a, b, c)$ and $(\dot{a}, \dot{b}, \dot{c})$ be as above. There are three integers $p_{0}, p_{1}$ and $p_{\infty}$ satisfying $p_{0}+p_{1}+p_{\infty} \in 2 \mathbf{Z}$ such that the exponents of the two equations are related as follows:

$$
1-c=1-\dot{c}+p_{0}, \quad c-a-b=\dot{c}-\dot{a}-\dot{b}+p_{1}, \quad b-a=\dot{b}-\dot{a}+p_{\infty} .
$$

The chart is denoted by $\mathbf{X}\left(p_{0}, p_{1}, p_{\infty}\right)$. In the following sections, we illustrate $\mathbf{X}$.

2. Result: Illustration of the chart $\mathbf{X}\left(p_{0}, p_{1}, p_{\infty}\right)$. Throughout this paper, we put $\infty$ at the top of the chart, and the arc $\overline{01}$ at the bottom.

2.1. $p_{0}, p_{1}, p_{\infty} \geq 0$. In this subsection, we assume $p_{0}, p_{1}, p_{\infty} \geq 0$. We first draw the chart when

$$
p_{0}+p_{1} \geq p_{\infty}, \quad p_{\infty}+p_{0} \geq p_{1}, \quad p_{1}+p_{\infty} \geq p_{0},
$$

and next draw the chart when one of the $p_{i}$ 's is greater than the sum of the other two; without loss of generality, we assume $p_{0}+p_{1}<p_{\infty}$.

2.1.1. $p_{0}+p_{1} \geq p_{\infty}, p_{\infty}+p_{0} \geq p_{1}, p_{1}+p_{\infty} \geq p_{0}$. By the assumption on the $p_{i}$ 's, there are integers $r_{0}, r_{1}, r_{\infty} \geq 0$ in a unique way satisfing

$$
p_{0}=r_{1}+r_{\infty}, \quad p_{1}=r_{\infty}+r_{0}, \quad p_{\infty}=r_{0}+r_{1}
$$

actually they are given by

$$
r_{0}=\frac{-p_{0}+p_{1}+p_{\infty}}{2}, r_{1}=\frac{p_{0}-p_{1}+p_{\infty}}{2}, r_{\infty}=\frac{p_{0}+p_{1}-p_{\infty}}{2} .
$$

The chart $\mathbf{X}\left(p_{0}, p_{1}, p_{\infty}\right)$ consists of $D$ and three parts $Y_{0}\left(r_{0}\right), Y_{1}\left(r_{1}\right)$ and $Y_{\infty}\left(r_{\infty}\right)$ surrounding $D$ as in the left of Figure 5. The right hand sides of Figures 5 and 6 show enlargements of $Y_{\infty}\left(r_{\infty}\right)$ for $r_{\infty}=0,1,2, \ldots$ For instance, $Y_{\infty}(0)$ is just empty, $Y_{\infty}(1)$ consists of $C, \bar{A}, \bar{D}$ and $\bar{B}$ (see Figure 12), and $Y_{\infty}(2)$ is shown in Figure 13. For later convenience, the corresponding diagram is also shown on the left of Figure 6 . There, two letters representing two triangles are connected by a segment if they have an edge in common.

Cyclic changes

$$
A \rightarrow B \rightarrow C \rightarrow A
$$

take $Y_{\infty}$ to $Y_{0}$ and then to $Y_{1}$. 


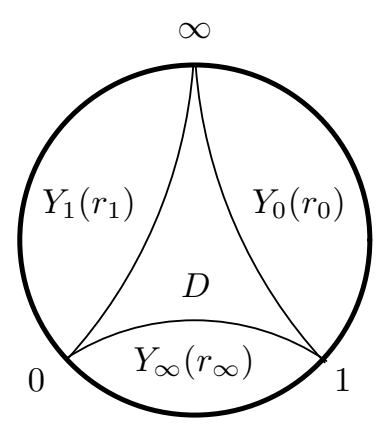

X

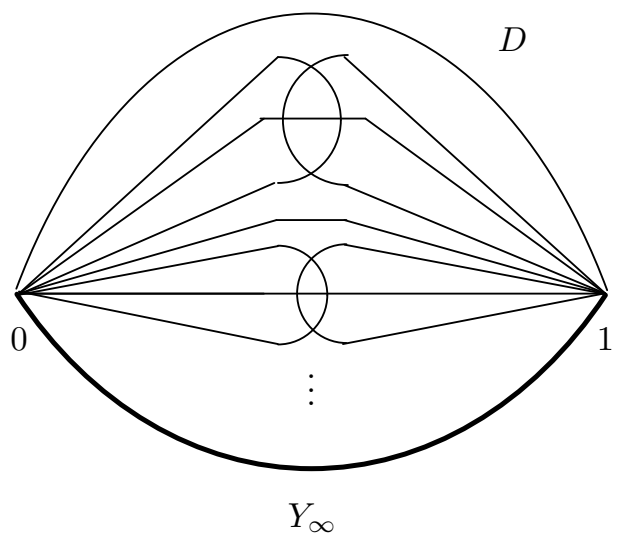

Fig. 5. $Y_{0}, Y_{1}, Y_{\infty}$ and $D$ forming $\mathbf{X}$, contents of $Y_{\infty}$
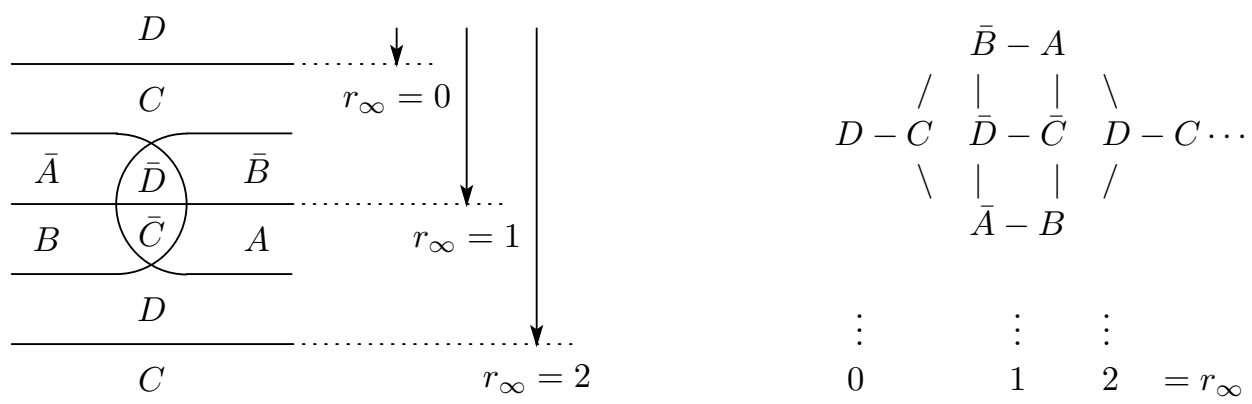

Fig. 6. Enlargement of $Y_{\infty}=Y_{\infty}\left(r_{\infty}\right)$, and its diagram

2.1.2. $p_{0}+p_{1}<p_{\infty}$. The chart $\mathbf{X}\left(p_{0}, p_{1}, p_{\infty}\right)$ can be made from $\mathbf{X}\left(p_{0}, p_{1}, p_{0}+p_{1}\right)$, which consists of $Y_{1}\left(p_{1}\right), D$ and $Y_{0}\left(p_{0}\right)$. We add some curves in the $D$-part starting from $\infty$ to make a vertical stripe

$$
D-A-\bar{C}-B
$$

$p_{\infty}-p_{0}-p_{1}$ times as in Figure 7 . The simplest case $\mathbf{X}(0,0,2)$ is shown in Figure 16.
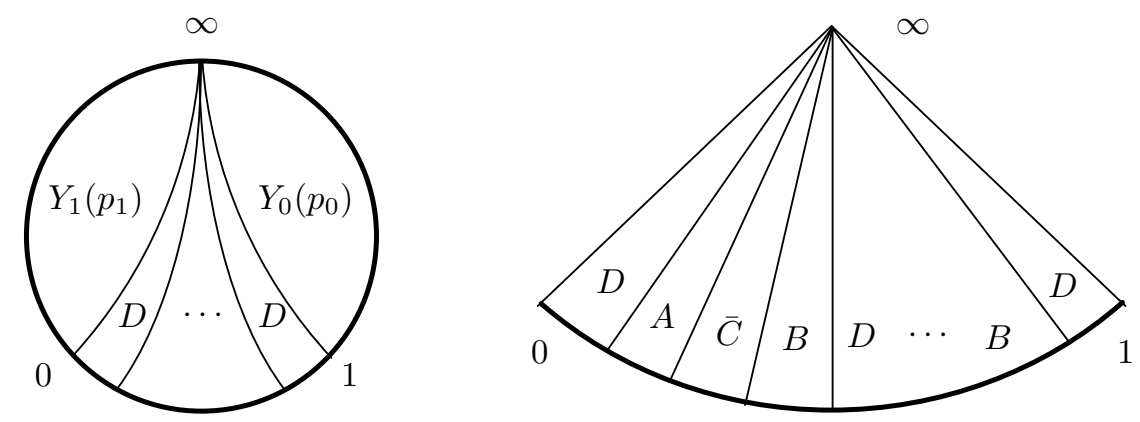

Fig. 7. $p_{0}+p_{1}<p_{\infty}$ : stripe sandwiched by $Y_{1}$ and $Y_{0}$ 
2.2. When two of the three are negative. If, say, $p_{0}$ and $p_{1}$ are negative, then the chart $\mathbf{X}\left(p_{0}, p_{1}, p_{\infty}\right)$ is homeomorphic to $\mathbf{X}\left(q_{0}, q_{1}, q_{\infty}\right)$, where $q_{0}=-p_{0}-1, q_{1}=-p_{1}-1, q_{\infty}=$ $p_{\infty}$

2.3. When one of the three is negative. If, say, $p_{0}<0$, consider the triple $\left(q_{0}, q_{1}, q_{\infty}\right)$ of non-negative integers, where $q_{0}=-p_{0}-1, q_{1}=p_{1}, q_{\infty}=p_{\infty}$. The chart $\mathbf{X}\left(p_{0}, p_{1}, p_{\infty}\right)$ will be denoted by $\mathbf{X}\left[q_{0}, q_{1}, q_{\infty}\right]$. Since the sum of the three $q$ 's is now odd, the sum of two $q$ 's cannot be equal to the third. We first draw the chart when

$$
q_{0}+q_{1}>q_{\infty}, \quad q_{\infty}+q_{0}>q_{1}, \quad q_{1}+q_{\infty}>q_{0},
$$

and next draw the chart when one of the $q_{i}$ 's is greater than the sum of the other two; without loss of generality, we assume $q_{0}+q_{1}<q_{\infty}$.

In any case, the vertices $s(0), s(1)$ and $s(\infty)$ are ordered negatively around $D$ (see Figure 8).
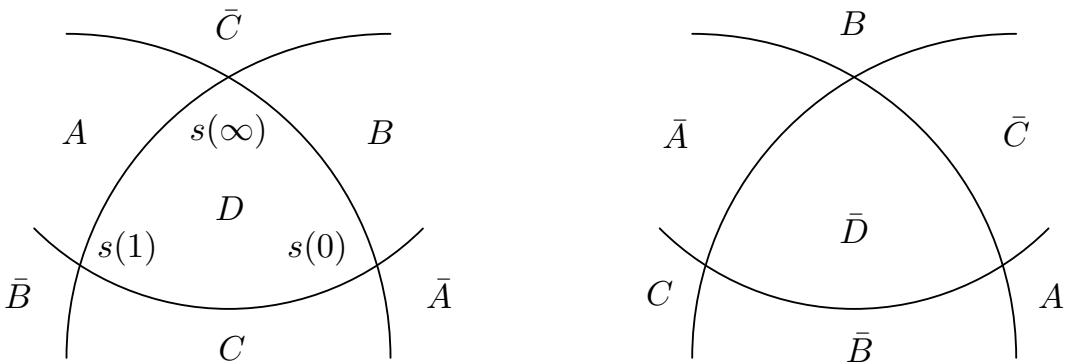

Fig. 8. $s(0), s(1)$ and $s(\infty)$ are ordered negatively around $D$

2.3.1. $q_{0}+q_{1}>q_{\infty}, q_{\infty}+q_{0}>q_{1}, q_{1}+q_{\infty}>q_{0}$. By the assumption on the $q_{i}$ 's, there are integers $r_{0}, r_{1}, r_{\infty} \geq 0$ in a unique way satisfying

$$
q_{0}=r_{1}+r_{\infty}+1, \quad q_{1}=r_{\infty}+r_{0}+1, \quad q_{\infty}=r_{0}+r_{1}+1 ;
$$

actually they are given by

$$
r_{0}=\frac{-q_{0}+q_{1}+q_{\infty}-1}{2}, r_{1}=\frac{q_{0}-q_{1}+q_{\infty}-1}{2}, r_{\infty}=\frac{q_{0}+q_{1}-q_{\infty}-1}{2} .
$$

The chart $\mathbf{X}\left[q_{0}, q_{1}, q_{\infty}\right]$ consists of the central part shown in Figure 9 and three parts $Z_{0}\left(r_{0}\right), Z_{1}\left(r_{1}\right)$ and $Z_{\infty}\left(r_{\infty}\right)$ surrounding the central part as in Figure 10.

The part $Z_{\infty}\left(r_{\infty}\right)$ is given by the following diagram (cf. Figure 6). Cyclic changes $A \rightarrow B \rightarrow C \rightarrow A$ take $Z_{\infty}$ to $Z_{0}$ and then to $Z_{1}$.

$$
\begin{aligned}
& \begin{array}{rll} 
& A-\bar{B} \\
C-D & \mid \bar{C}-\bar{D} \quad C-D \cdots \\
\backslash & |\quad| \quad / \\
& B-\bar{A}
\end{array} \\
& \begin{array}{lll}
0 & 1 & 2
\end{array}
\end{aligned}
$$




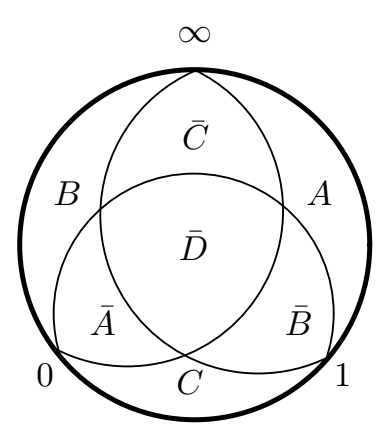

Fig. 9. $\mathbf{X}[111]$, the central part

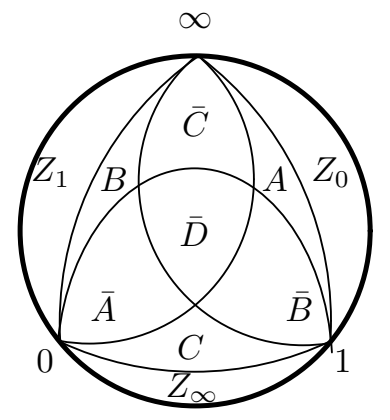

Fig. 10. Three parts $Z_{0}\left(r_{0}\right), Z_{1}\left(r_{1}\right)$ and $Z_{\infty}\left(r_{\infty}\right)$ surrounding the central part

2.3.2. $q_{0}+q_{1}<q_{\infty}$. The chart $\mathbf{X}\left[q_{0}, q_{1}, q_{\infty}\right]$ can be made from $\mathbf{X}\left[q_{0}, q_{1}, q_{0}+q_{1}+1\right]$, which consists of $Z_{1}\left(q_{1}\right), B-\bar{C}-A$ and $Z_{0}\left(q_{0}\right)$. We add some curves in the $\bar{C}$-part starting from $\infty$ to make a vertical stripe

$$
B-\bar{C}-A-D-\cdots-D-B-\bar{C}-A
$$

where $D$ appears $q_{\infty}-q_{0}-q_{1}-1$ times (see Figure 11 ). The simplest one $\mathbf{X}(0,0,1)$ is shown in Figure 17.
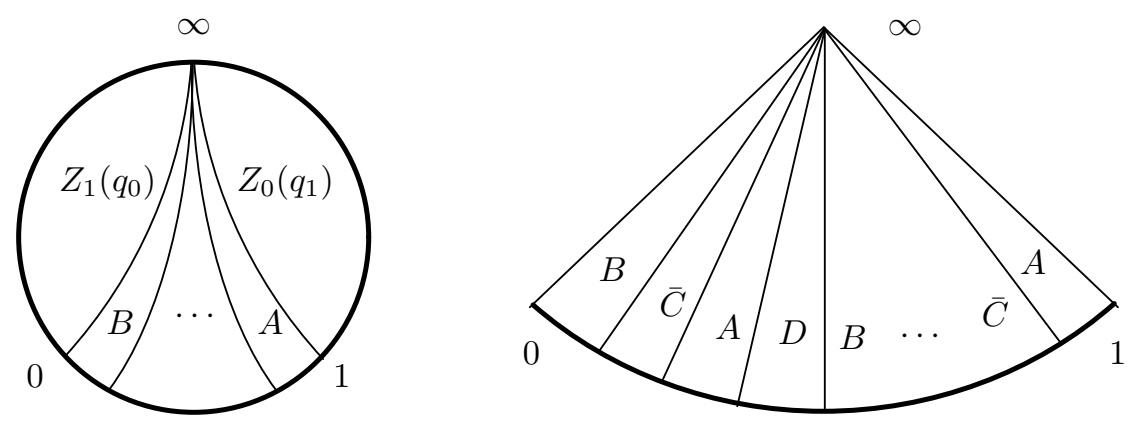

Fig. 11. $q_{0}+q_{1}<q_{\infty}$, stripe

2.4. When all the three p's are negative. Put

$$
q_{0}=-p_{0}-1, \quad q_{1}=-p_{1}-1, \quad q_{\infty}=-p_{\infty}-1 ;
$$

the chart $\mathbf{X}\left(p_{0}, p_{1}, p_{\infty}\right)$ is homeomorphic to the chart $\mathbf{X}\left[q_{0}, q_{1}, q_{\infty}\right]$, which is studied in the previous section.

3. Proof. We fix some notaion: For a real number $x$, there is a unique real number $\xi$ such that $x-\xi \in \mathbf{Z}$ and $0 \leq \xi<1$; this number $\xi$ will be denoted by modp $x$.

For a triple $(a, b, c)$ of real numbers, we put

$$
\lambda:=1-c, \quad \mu:=c-a-b, \quad \nu:=b-a .
$$


Exchanging $a$ and $b$ if necessary, there exists uniquely a triple $(\dot{a}, \dot{b}, \dot{c})$ of real numbers satisfying

$$
\dot{a}-a, \dot{b}-b, \dot{c}-c \in \mathbf{Z}
$$

such that

$$
\dot{\lambda}=\operatorname{modp} \lambda, \quad \dot{\mu}=\operatorname{modp} \mu, \quad \dot{\nu}=\operatorname{modp} \nu,
$$

where

$$
\dot{\lambda}:=1-\dot{c}, \quad \dot{\mu}:=\dot{c}-\dot{a}-\dot{b}, \quad \dot{\nu}:=\dot{b}-\dot{a} .
$$

We assume

$$
\dot{\lambda}, \dot{\mu}, \dot{\nu}>0 \text { and } \dot{\lambda}+\dot{\mu}+\dot{\nu}>1
$$

Define the three integers as

$$
p_{0}:=\lambda-\dot{\lambda}, \quad p_{1}:=\mu-\dot{\mu} \quad \text { and } \quad p_{\infty}:=\nu-\dot{\nu} .
$$

As in $\S 1$, for a triple of real numbers $(a, b, c)$ we define the three circles $C_{(-\infty, 0)}, C_{(0,1)}$ and $C_{(0, \infty)}$. In the same way, the triple $(\dot{a}, \dot{b}, \dot{c})$ defines the three circles $\dot{C}_{(-\infty, 0)}, \dot{C}_{(0,1)}$ and $\dot{C}_{(0, \infty)}$.

LEMma. There exists a linear fractional transformation which takes $C_{(-\infty, 0)}$ to $\dot{C}_{(-\infty, 0)}$, $C_{(0,1)}$ to $\dot{C}_{(0,1)}, C_{(0, \infty)}$ to $\dot{C}_{(0, \infty)}$, and $C_{(-\infty, 0)} \cup C_{(0,1)} \cup C_{(0, \infty)}$ to $\dot{C}_{(-\infty, 0)} \cup \dot{C}_{(0,1)} \cup \dot{C}_{(0, \infty)}$.

Proof. In general two intersecting circles determine two angles, say $\alpha$ and $\beta$, satisfying $0 \leq \alpha, \beta \leq \pi, \alpha+\beta=\pi$; so we say that two circles intersect with angles $\{\alpha, \beta\}$. The angles of the triangle $\dot{D}$ are $\{\dot{\lambda} \pi, \dot{\mu} \pi, \dot{\nu} \pi\}$. Note that the triangles $A$ and $\dot{\dot{A}}$ have angles $\{\dot{\lambda} \pi,(1-\dot{\nu}) \pi,(1-\dot{\mu}) \pi\}$, and so on. Since $p_{0}, p_{1}$ and $p_{\infty}$ are integers, the circles $C_{(-\infty, 0)}$ and $C_{(0,1)}$ intersect with angles $\{\dot{\lambda} \pi,(1-\dot{\lambda}) \pi\}$, the circles $C_{(0,1)}$ and $C_{(1, \infty)}$ intersect with angles $\{\dot{\mu} \pi,(1-\dot{\mu}) \pi\}$, and the circles $C_{(1, \infty)}$ and $C_{(-\infty, 0)}$ intersect with angles $\{\dot{\nu} \pi,(1-\dot{\nu}) \pi\}$. Since moreover $p_{0}+p_{1}+p_{\infty} \in 2 \mathbf{Z}$, any of the eight triangles cut out from the three circles $C_{(-\infty, 0)}$ etc. cannot have angles $\{(1-\dot{\lambda}) \pi,(1-\dot{\mu}) \pi,(1-\dot{\nu}) \pi\}$ or $\{(1-\dot{\lambda}) \pi, \dot{\mu} \pi, \dot{\nu} \pi\}$ or $\{\dot{\lambda} \pi,(1-\dot{\mu}) \pi, \dot{\nu} \pi\}$ or $\{\dot{\lambda} \pi, \dot{\mu} \pi,(1-\dot{\nu}) \pi\}$. Thus these eight triangles have the same angles with the eight triangles cut out from the three circles $\dot{C}_{(-\infty, 0)}$ etc.

3.1. When $p_{0}, p_{1}, p_{\infty} \geq 0$. We have

$$
\operatorname{modp}|\lambda|=\dot{\lambda}, \quad \operatorname{modp}|\mu|=\dot{\mu}, \quad \operatorname{modp}|\nu|=\dot{\nu} .
$$

Though this is quite obvious, thanks to this fact, the three angles modulo $\pi$ of the Schwarz triangle $T$ are the same as those of $\dot{T}$, and so we have $D=\dot{D}$. We consider the map

$$
f:=s \circ \dot{s}^{-1}: D \rightarrow T \subset Z,
$$

and see how $T$ overflows, under $f$, from $D$.

(1) When $\mathbf{p}:=\left(p_{0}, p_{1}, p_{\infty}\right)=(1,1,0)$, then $T$ overflows from $D$ through the side $\overline{s(0) s(1)}$ of $D$, and the image $T$ is bounded by the complementary arc $C_{01}-\overline{s(0) s(1)}$, as is shown in the right of Figure 12. Note that the angle of $T$ at $s(0)$ is greater than that of $D$ by $\pi$.

When $\mathbf{p}=(2,2,0)$, then the angle of $T$ at $s(0)$ is greater than that of $D$ by $2 \pi$. Though $T$ is bounded by the three sides of $D, D$ is doubly covered as is shown in 13 . When $\mathbf{p}(r, r, 0)(r \geq 0), T$ overflows the side $\overline{s(0) s(1)}$ as above, and the angle of $T$ at $s(0)$ 


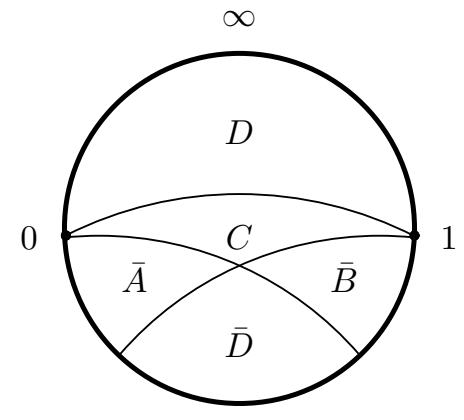

X

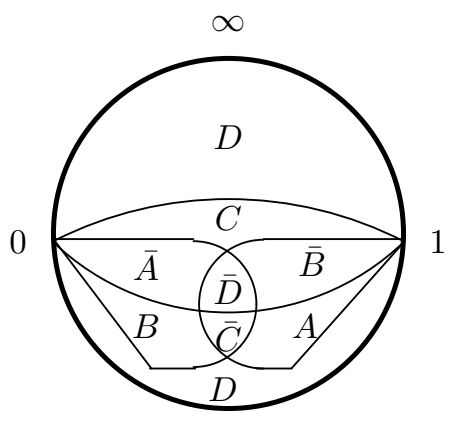

X

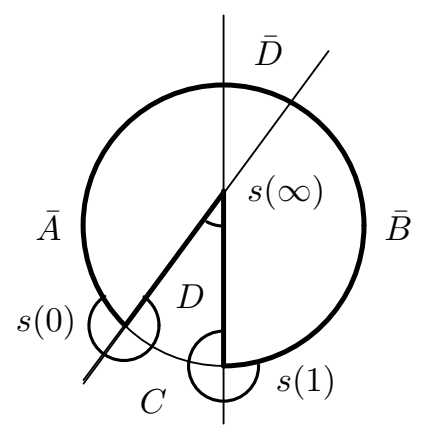

T

Fig. 12. $\mathbf{X}(1,1,0)$

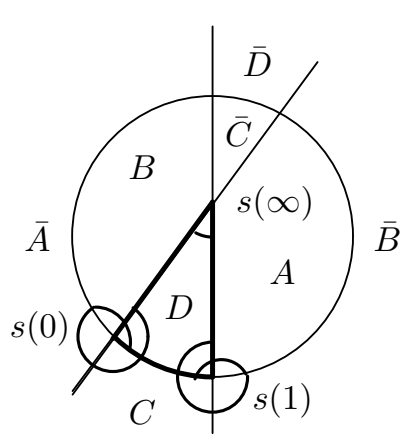

T

Fig. 13. $\mathbf{X}(2,2,0)$

is greater than that of $D$ by $r \pi$. Now we can readily see that $\mathbf{X}(r, r, 0)$ consists of $D$ and $Y_{\infty}(r)$ as is illustrated in Figure 5.

(2) When $\mathbf{p}=\left(p_{0}, p_{1}, p_{0}+p_{1}\right)$, since $\mathbf{p}=\left(p_{0}, 0, p_{0}\right)+\left(0, p_{1}, p_{1}\right)$, the image $T$ overflows through the sides $\overline{s(\infty) s(0)}$ and $\overline{s(1) s(\infty)}$ of $D$. The situation explained in (1) for the side $\overline{s(0) s(1)}$ occurs doubly along the other two sides. A typical example $\mathbf{X}(1,1,2)$ is shown in Figure 14.

(3) When

$$
p_{0}+p_{1}>p_{\infty}, \quad p_{\infty}+p_{0}>p_{1}, \quad p_{1}+p_{\infty}>p_{0}
$$

we have

$$
\mathbf{p}=\left(0, r_{0}, r_{0}\right)+\left(r_{1}, 0, r_{1}\right)+\left(r_{\infty}, r_{\infty}, 0\right),
$$

where $p_{0}=r_{1}+r_{\infty}, p_{1}=r_{\infty}+r_{0}, p_{\infty}=r_{0}+r_{1}$. Thus the image $T$ overflows through the three sides of $D$, and the situation explained in (1) for the side $\overline{s(0) s(1)}$ occurs triply along the three sides. A typical example $\mathbf{X}(2,2,2)$ is shown in Figure 15.

(4) When $p_{0}+p_{1}<p_{\infty}$, we have

$$
\mathbf{p}=\left(p_{0}, 0, p_{0}\right)+\left(0, p_{1}, p_{1}\right)+(0,0,2 r), \quad r=\left(p_{\infty}-p_{0}-p_{1}\right) / 2 .
$$




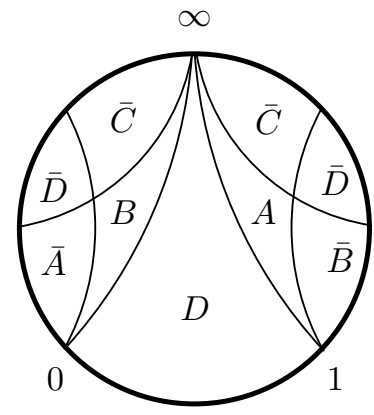

Fig. 14. $\mathbf{X}(1,1,2)$

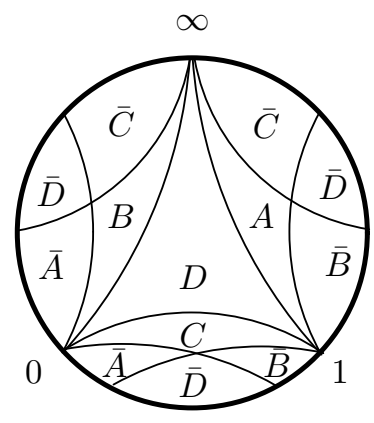

Fig. 15. $\mathbf{X}(2,2,2)$

As is shown in Figure 16, when $\mathbf{p}=(0,0,2)$, the image $T$ is just an extension of $D$ around the vertex $s(\infty)$ by $2 \pi$. So the chart $\mathbf{X}(0,0,2)$ is given by the diagram $D-A-\bar{C}-B-D$. In this way, the chart $\mathbf{X}(0,0,2 r)$ is an extension of $D$ around the vertex $s(\infty)$ by $2 r \pi$; its corresponding diagram is $r$ times $D-A-\bar{C}-B-D$, that is

$$
D-A-\bar{C}-B-D-\cdots-D-A-\bar{C}-B-D \text {. }
$$

The chart $\mathbf{X}(\mathbf{p})$ is obtained by replacing the left extreme $D$ of $\mathbf{X}(0,0,2 r)$ by $Y_{1}\left(p_{0}\right)$ and the right extreme $D$ of $\mathbf{X}(0,0,2 r)$ by $Y_{0}\left(p_{1}\right)$.

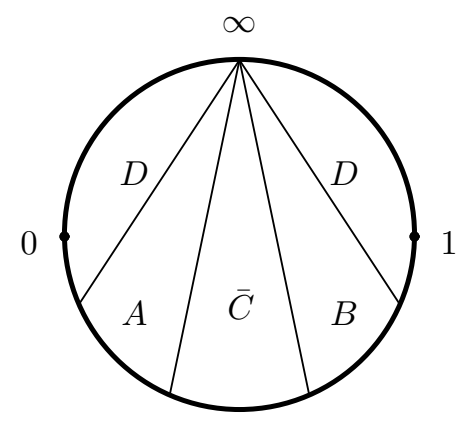

$X$

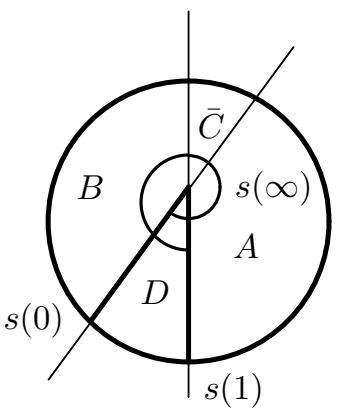

T

Fig. 16. $\mathbf{X}(0,0,2)$

3.2. Otherwise. We define the non-negative integers $q_{0}, q_{1}$ and $q_{\infty}$ by

$$
q_{i}:=\left\{\begin{array}{cl}
p_{i} & \text { if } p_{i} \geq 0, \\
1-p_{i} & \text { if } p_{i}<-1,
\end{array} \quad i=0,1, \infty .\right.
$$

If for example $p_{0}$ is negative, then we have

$$
\operatorname{modp}|\lambda|=1-\dot{\lambda}, \quad \text { and } \quad|\lambda|=\left|\dot{\lambda}+p_{0}\right|=1-\dot{\lambda}+q_{0},
$$

which just implies that, at the vertex $s(0)$, the image $T$ has angle $\left(1-\dot{\lambda}+q_{0}\right) \pi$. Note that the parity of $q_{0}+q_{1}+q_{\infty}$ matters. If $q_{0}+q_{1}+q_{\infty}$ is even, that is, if two of the $p_{i}$ 's 
are negative, then nothing new happens; it reduces to the case treated in the previous section: the chart $\mathbf{X}\left(p_{0}, p_{1}, p_{\infty}\right)$ is homeomorphic to $\mathbf{X}\left(q_{0}, q_{1}, q_{\infty}\right)$. (Note however that $D$ is equal to $\dot{A}, \dot{B}$ or $\dot{C}$.) If $q_{0}+q_{1}+q_{\infty}$ is odd, that is, if one or all of the $p_{i}$ 's are negative, then we must study the chart anew. We denote $\mathbf{X}\left(p_{0}, p_{1}, p_{\infty}\right)$ by $\mathbf{X}\left[q_{0}, q_{1}, q_{\infty}\right]$.

(1) We first study the simplest one $\mathbf{X}(0,-1,1)=\mathbf{X}[0,0,1]$. Since $p_{0}=0$, the map $\dot{s}$ and $s$ are locally isomorphic around $z=0$, so the images $\dot{T}$ around $\dot{s}(0)$ and $T$ around $s(0)$ are locally isomorphic. Since $p_{1}=-1$, though $q_{0}=0$, the angle of $\dot{T}$ at $\dot{s}(1)$ and that of $T$ at $s(1)$ are complementary. Since $p_{\infty}=1$, the angle of $T$ at $s(\infty)$ is greater than of $\dot{T}$ by $\pi$. This argument is illustrated in Figure 17. The vertex $s(1)$ is the intersection of the two circles $C_{01}$ and $C_{1 \infty}$ other than $\dot{s}(1)$. Since $D$ is defined as the triangle with vertices $\{s(0), s(1), s(\infty)\}$, the triangle $\dot{B}$ is called $D$, the triangle $\dot{D}$ is called $B$, and so on.

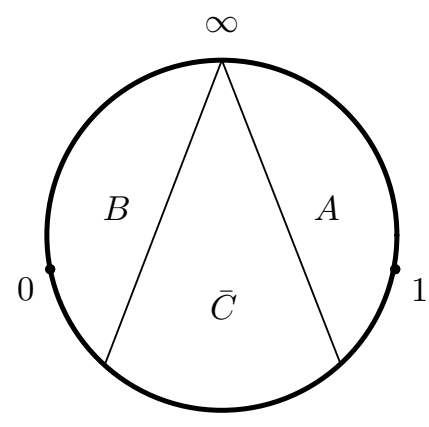

$X$

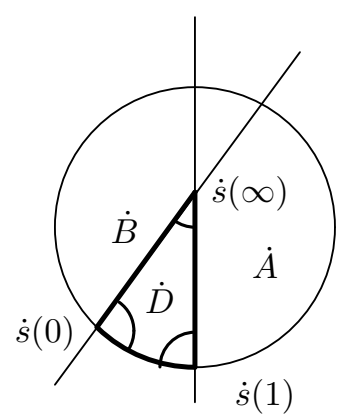

$\dot{T}$

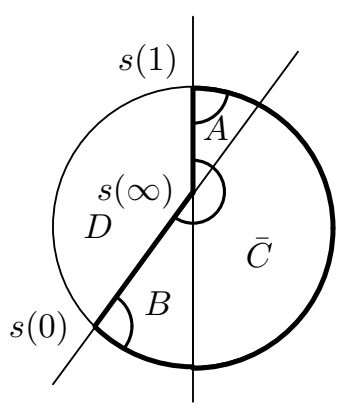

$T$

Fig. 17. $\mathbf{X}(0,-1,1)=\mathbf{X}[0,0,1]$

When $q_{0}+q_{1}<q_{\infty}$, since

$$
\mathbf{q}:=\left(q_{0}, q_{1}, q_{\infty}\right)=\left(q_{0}, 0, q_{0}\right)+\left(0, q_{1}, q_{1}\right)+(0,0,2 r)+(0,0,1)
$$

where $r=\left(q_{\infty}-q_{0}-q_{1}-1\right) / 2$, the chart $\mathbf{X}[\mathbf{q}]$ is made from $\mathbf{X}[0,0,1]$ by putting $Z_{1}\left(q_{0}\right)$ on the left, $Z_{0}\left(q_{1}\right)$ on the right, and put $r$ times the stripe $B-\bar{C}-A-D$. For example, the chart $\mathbf{X}[0,1,2]$ is shown in Figure 18 .

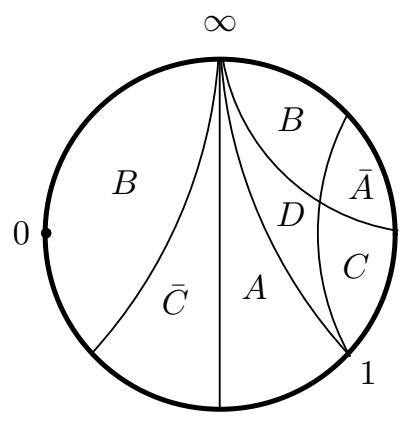

Fig. 18. $\mathbf{X}[0,1,2]$ 
(2) The chart $\mathbf{X}[1,1,1]$ is already shown in Figure 9. When

$$
q_{0}+q_{1}>q_{\infty}, \quad q_{\infty}+q_{0}>q_{1}, \quad q_{1}+q_{\infty}>q_{0},
$$

since

$$
\mathbf{q}=\left(0, r_{0}, r_{0}\right)+\left(r_{1}, 0, r_{1}\right)+\left(r_{\infty}, r_{\infty}, 0\right)+(1,1,1),
$$

the chart $\mathbf{X}[\mathbf{q}]$ is obtained from $\mathbf{X}[1,1,1]$ by putting $Z_{0}\left(r_{0}\right)$ on the right, $Z_{1}\left(r_{1}\right)$ on the left, and $Z_{\infty}\left(r_{\infty}\right)$ at the bottom, as is shown in Figure 10.

\section{References}

[OY] H. Ochiai and M. Yoshida, Polynomials associated with the hypergeometric functions with finite monodromy groups, Internat. J. Math. 15 (2004), 629-649.

[Yo] M. Yoshida, Hypergeometric Functions, My Love: Modular Interpretations of Configuration Spaces, Vieweg Verlag, Wiesbaden, 1997. 